05

\title{
Пространственные характеристики сверхпроводников и 3D-холловский микроскоп для их исследования
}

\author{
(C) X.P. Ростами \\ Фрязинский филиал Института радиотехники и электроники им. В.А. Котельникова РАН, \\ 141190 Фрязино, Московская обл., Россия \\ e-mail: rostami@ms.ire.rssi.ru
}

Поступило в Редакцию 23 июля 2018 г.

В окончательной редакции 27 мая 2020 г.

Принято к публикации 2 июня 2020 г.

На основе преобразователей Холла разработан растровый микроскоп с площадью обзора $5 \times 5 \mathrm{~mm}$ и с его помощью с высокими пространственным разрешением и чувствительностью измерена топология магнитного поля $B(X, Y, Z)$ на поверхности и вокруг образцов $\mathrm{YBa}_{2} \mathrm{Cu}_{3} \mathrm{O}_{7-x}$ и $\mathrm{Bi}_{2} \mathrm{Sr}_{2} \mathrm{CaCu}_{2} \mathrm{O}_{8+x}$ без применения формул электродинамики. Дифференциальная методика с фиксацией начала и конца перемещения датчика обеспечивала точность сканирования по осям $X Y, Z \sim 10$ и $\sim 1 \mu \mathrm{m}$ соответственно в грубом и плавном диапазонах. Максимальный размер перемещения по оси $Z$ составлял $25 \mathrm{~mm}$. Для устранения ошибок измерения нормированные распределения $B(Z) / B^{\max }(0)$ были экстраполированы к оси ординат, смещенной по оси $Z$ на величину зазора между поверхностью образца и преобразователем Холла.

Ключевые слова: $3 D$-холловский микроскоп, ВТСП, двойникование, преобразователь Холла.

DOI: 10.21883/JTF.2020.12.50123.291-18

\section{Введение}

Известно, что физические свойства высокотемпературных сверхпроводников (ВТСП) очень чувствительны к незначительным пространственным неоднородностям, так как их длина когерентности является величиной одного порядка с межатомными и межэлектронными расстояниями $[1,2]$. Было обнаружено, что даже самые совершенные монокристаллы $\mathrm{YBa}_{2} \mathrm{Cu}_{3} \mathrm{O}_{7-x}$ (YBCO) coдержат большое количество регулярно расположенных границ двойникования (ГД) с плоскостью двойникования (110). В зависимости от способа приготовления материала, ГД могут иметь ширину $\Delta d \sim 30-50 \AA$, а расстояние $d$ между ГД может составлять $\sim 200-2000 \AA[1-6]$. Наличие ГД присуще самой природе ҮВСО ВТСП, так как двойниковые домены образуются в результате структурного перехода из тетрагональной в орторомбическую фазу при температуре $\sim 700^{\circ} \mathrm{C}[1-6]$; следовательно, от ГД избавиться не удается, и с ними приходится считаться. Выяснение степени неоднородности ВТСП образцов с помощью исследования особенностей проникновения (выхода), захвата магнитного потока (ЗМП) и пространственного распределения параметров ЗМП в таких средах имеет большое научное и прикладное значение (см. [7] и приведенную там литературу). Использование для наблюдения картины распределения ЗМП микроскопов на основе пьезопреобразователей с верхним пределом микроперемещений в несколько десятков $\mu \mathrm{m}$ вызывает определенные трудности, требуя сложных схем коррекции из-за гистерезиса и нелинейности перемещения [8]. Холловские микроскопы [7,9-11] непосредственно измеряют индукцию поля и позволяют с высокой линейностью визуализировать магнитное мик- росостояние протяженных объектов. Однако они имеют сравнительно низкие пространственное разрешение и чувствительность к регистрируемому сигналу, что не позволяет отчетливо выделить спектры сигналов отклика объектов на слабое магнитное поле. Разработанные к настоящему времени микроскопы прецизионно измеряют в основном двумерную картину распределения поля, а для построения трехмерной картины применяются модели и программы с использованием формул электродинамики. Корректное построение $3 D$-картины удается осуществить лишь для структур с ранее известной топологией. В большинстве случаев распределение внутренних полей и магнитного отклика образцов приходится выяснять. Например, при исследовании пространственных распределений параметров неоднородных полей, полей размагничивания поликристаллических образцов, где размеры, форма, взаиморасположение и ориентация кристаллитов заранее неизвестны, $3 D$-картину распределения полей можно получить только с помощью непосредственных измерений [7]. Кроме того, для исключения гистерезиса при прямом и обратном перемещении координатных столиков очень важно точно фиксировать начальные точки перемещения. Решение этой задачи очень востребовано для точного составления полной картины распределения магнитного поля протяженных объектов путем сшивания более точно измеренных полей мелкомасштабных участков поверхности образца [8]. Исходя из вышеизложенного, разработка высокочувствительного низкотемпературного трехмерного растрового холловского микроскопа, позволяющего проводить исследование объектов одновременно в однородных и осциллирующих затухающих локальных магнитных полях 
с высокой линейностью и пространственным разрешением, представляется актуальной задачей.

\section{1. Конструкция и принцип действия микроскопа}

На рис. 1 приведена структурная схема системы позиционирования микроскопа, на рис. 2 - сборная конструкция системы позиционирования микроскопа с изображением омических контактов. Закреплением образца на координатный столик устанавливается соответствие между перемещениями $X, Y, Z$ и соответствующими напряжениями потенциометров позиционирования следующим способом. Сначала при настройке механической части установки обеспечивается минимальное расстояние в $1 \pm 0.1 \mu \mathrm{m}$ между строго параллельными, тщательно отполированными поверхностями направляющей оправы с образцом 2 и медного диска 3 , в котором установлены преобразователи Холла (ПХ). Такое расстояние задавалось следующим образом. Вначале подложка из $\mathrm{NdGaO}_{3}$ толщиной $1 \mathrm{~mm}$ устанавливалась в оправе 12 (рис. 1), внутри которой имелась выемка глубиной $1 \mathrm{~mm}$, а затем оправа с подложкой устанавливались внутри направляющей 18 и фиксировались с помощью винтика 17.

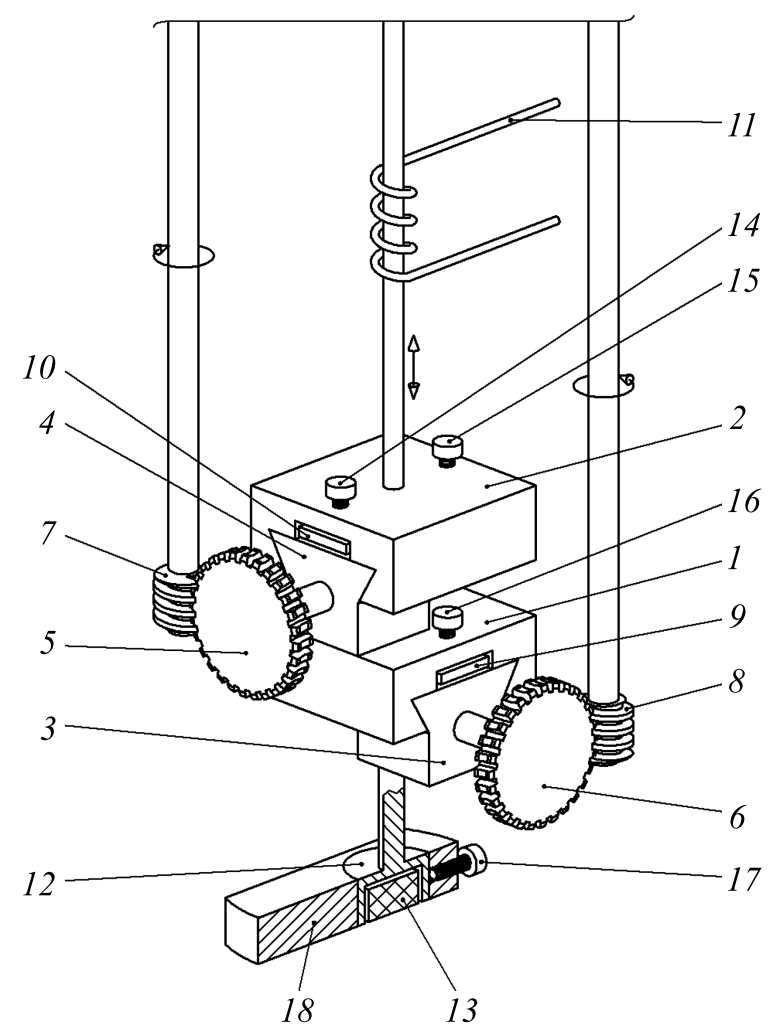

Рис. 1. Структурная схема системы позиционирования микроскопа. 1,2 - $X$-, $Y$-платформы; $3,4-$ ходовые гайки; 5, 6 - червячные колеса; 7,8 - червяки; 9, $10-$ регулирующие пластины; 11 - нагреватель; 12 - оправа; 13 - образец, $14-16$ - регулирующие винтики; 17 - зажим; $18-$ направляющая оправы с образцом.

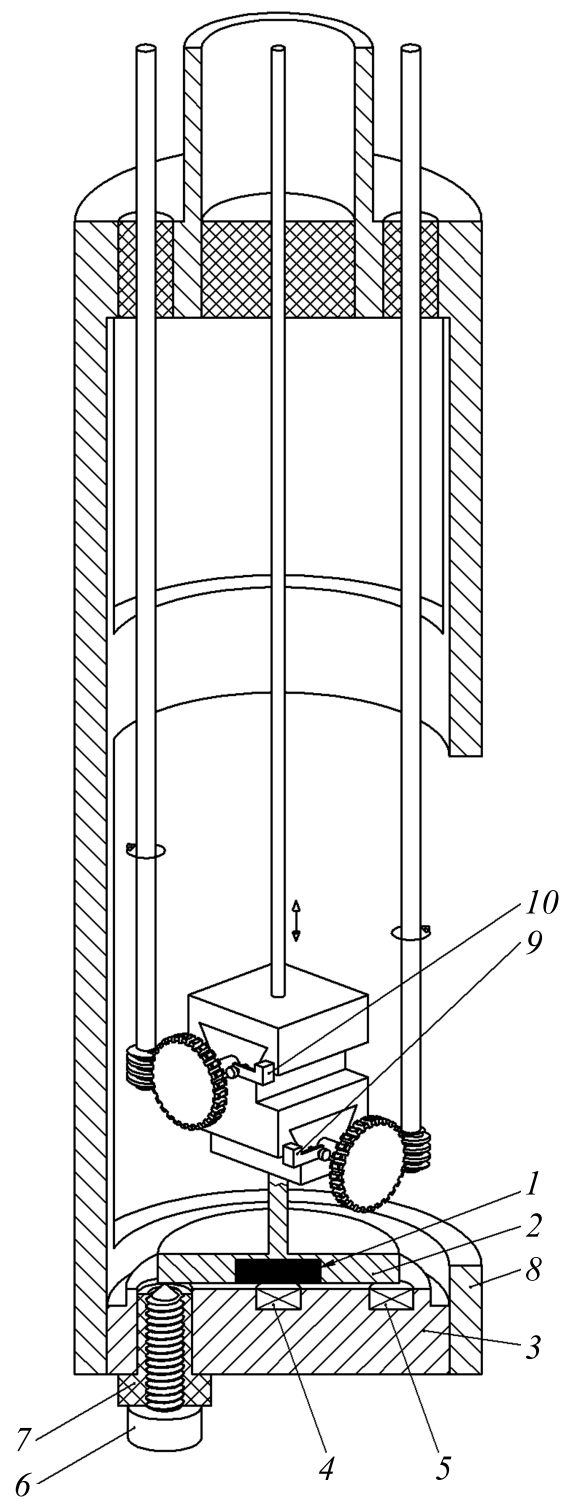

Рис. 2. Сборная конструкция системы позиционирования микроскопа с изображением омических контактов. 1 - образец; 2 - направляющая оправы с образцом; 3 - медный диск; 4, 5 - образователи Холла; 6 - латунный винт; 7 - тефлоновый изолятор; $8-$ латунный цилиндр; 9, $10-X$-, $Y$-омические контакты (такие же контакты имеются с боковых сторон).

Далее с помощью $Z$-перемещения обеспечивалось касание винтиком 6 направляющей оправы с образцом 18 (рис. 1). Винтик 6 , создающий омический контакт с направляющей оправы с образцом 2 , ввинчивался через изолятор 7 в медном диске 3. Затем после фиксации разрыва контакта, что соответствовало минимальному зазору между медным диском 3 и направляющей оправы с образцом 2 (рис. 2), с помощью дифференциального микровинта $Z$-перемещения направляющая оправы с образцом 2 отодвигалась на $2 \mu \mathrm{m}$. После этого ввинчиванием винтика 6 устанавливался омический контакт с направляющей оправы с подложкой 2. Далее направ- 
ляющая оправы с образцом 2 отодвигалась на $10 \mathrm{~mm}$, и установленная подложка заменялась подложкой из $\mathrm{NdGaO}_{3}$ толщиной $1 \mathrm{~mm}$, на поверхность которой лазерным распылением была нанесена эпитаксиальная пленка YВСО толщиной $\sim 1 \mu \mathrm{m}$. После этого осуществлялось $Z$-перемещение направляющей оправы с образцом 2 к винтику 6 до фиксации омического контакта. Затем винтик 6 отвинчивался до разрыва контакта, после чего проводилось измерение. Размеры и толщина зеркально отполированной с обеих сторон подложки задавались заранее, исходя из технологических требований напыления пленок. Медная оправа с углублением строго по толщине подложки также позволяла выполнить замену подложки на пленку с боковой стороны, не меняя заранее установленный зазор между образцом и ПХ. Кроме того, толщины подложки и образца дополнительно измерялись с помощью микроскопа. Для этого сначала с помощью двух омических контактов по оси $Z$ (сверху и снизу) устанавливалась толщина подложки (один из винтиков фиксировался, а другой регулировался). Точность зазора $2 \mu \mathrm{m}$ контролировалась также с помощью экстраполяции кривой $B(Z) / B^{\max }(0)$ к оси ординат, смещенной по оси $Z$ на $2 \mu \mathrm{m}$. Такую процедуру настройки необходимо проводить перед измерениями и для образцов другой толщины. Это позволяет компенсировать люфты резьбы микровинта при прямом и обратном перемещениях образца по оси Z. Чтобы уменьшить усилие вращающих механизмов, способное привести к деформации винтиков при детектировании омических контактов, сопротивление контактов регистрировалось с помощью высокочувствительного преобразователя сопротивление-напряжение. С помощью омических контактов таким же образом задавались и контролировались начало и конец перемещения $(X, Y)$ столиков. Перемещение столиков в трех направлениях регулировалось дифференциальными микровинтами, редукторами и вращающими механизмами с точностью 10 и $1 \mu \mathrm{m}$ соответственно в грубом и плавном диапазонах. Вращающие механизмы, управляющие работой червячных передач, подключены к синхронным двигателям СД-54, которые имели скорость $60 \mathrm{rev} / \mathrm{min}$ и позволяли путем переключения емкости менять направление вращения. Работа двигателей управлялась с помощью электроники путем фиксации начала и конца перемещения в направлениях $X, Y, Z$ по сигналу от омических контактов. Это позволяло существенно повысить точность перемещения в трех направлениях независимо от размера рабочей поверхности датчика.

Таким образом, после окончания сканирования по оси $X$ вводится новое значение координаты $Y$, для него повторяются измерения, и данные автоматически считываются компьютером. В результате снимается двухмерная картина распределения поля над образцом. Далее образец по заданному шагу перемещается по оси $Z$ вверх, и заново снимается распределение поля над образцом и т.д.
Построение $B(X), B(Y)$ и $B(Z)$ в прямом и обратном направлениях позволяли по показаниям ПХ определять степени гистерезиса и параллельности сканирования образца по отношению к поверхности ПХ. Для построения нормированных осевых распределений $B(X) / B(0)$, $B(Y) / B(0)$ и $B(Z) / B(0)$ от координат $X, Y, Z$ кубик малого размера с тремя одинаковыми по техническим характеристикам ПХ на трех взаимно перпендикулярных гранях закреплялся на трехкоординатный столик микроскопа. Таким образом измерялись компоненты $B_{x}, B_{y}, B_{z}$, величина $B=\left(B_{x}^{2}+B_{y}^{2}+B_{z}^{2}\right)^{1 / 2}$ и тензоры магнитного поля произвольного направления:

$$
B_{x, y, z}=\left|\begin{array}{lll}
\frac{\partial B_{x}}{\partial x} & \frac{\partial B_{x}}{\partial y} & \frac{\partial B_{x}}{\partial z} \\
\frac{\partial B_{y}}{\partial x} & \frac{\partial B_{y}}{\partial y} & \frac{\partial B_{y}}{\partial z} \\
\frac{\partial B_{z}}{\partial x} & \frac{\partial B_{z}}{\partial y} & \frac{\partial B_{z}}{\partial z}
\end{array}\right| .
$$

Магнитный момент образцов измерялся с помощью двух близких по техническим характеристикам ПХ. Один из ПХ находился под образцом, а опорный ПХ - в невозмущенном образцом магнитном поле. Каждый раз перед измерениями после задания необходимой температуры сначала образец перемещался по оси $Z=25 \mathrm{~mm}$ и только потом с помощью электронной схемы компенсировался сигнал разбаланса ПХ.

\section{2. Характеристики микроскопа}

Испытания показали, что благодаря использованию фиксаторов начала и конца перемещения столиков и применения принципа дифференциального перемещения разрешение системы позиционирования по трем координатам было лучше $1 \mu \mathrm{m}$. В [11] программноуправляемая система перемещения станка ЕР2006Н (фирма Everprecision, Тайвань) обеспечивала точность позиционирования $\sim 5 \mu \mathrm{m}$ по осям $X, Y$ и $\sim 9 \mu \mathrm{m}$ по оси $Z$ при комнатной температуре. С целью достижения более высокого пространственного разрешения $3 \mu \mathrm{m}$ автору пришлось промоделировать и корректировать показания ПХ для образца с ранее известной топологией. Чувствительность предлагаемого микроскопа по магнитному полю составляет $\sim 2.5 \cdot 10^{-3} \mathrm{G} / \mathrm{Hz}^{1 / 2}[12]$. Для оценки чувствительности микроскопа сначала с помощью откалиброванного ПХ на сравнительно большом токе через соленоид устанавливалась постоянная катушки, а затем при известном малом токе определялась величина поля и магнитная чувствительность микроскопа. Динамический диапазон предложенного микроскопа по магнитному полю составляет $\pm(1-3) \cdot 10^{3} \mathrm{G}$, а по температуре - $(2-300) \mathrm{K}$. Стабильность величин заданного магнитного поля и температуры лучше, чем $10-5 \mathrm{G}$ и $10^{-2} \mathrm{~K}$ соответственно. Известно, что слабые магнитные поля величиной $\sim 10^{-4}-10^{-3} \mathrm{G}$ проникают в керамические ВТСП $[13,14]$. Как показали исследования 

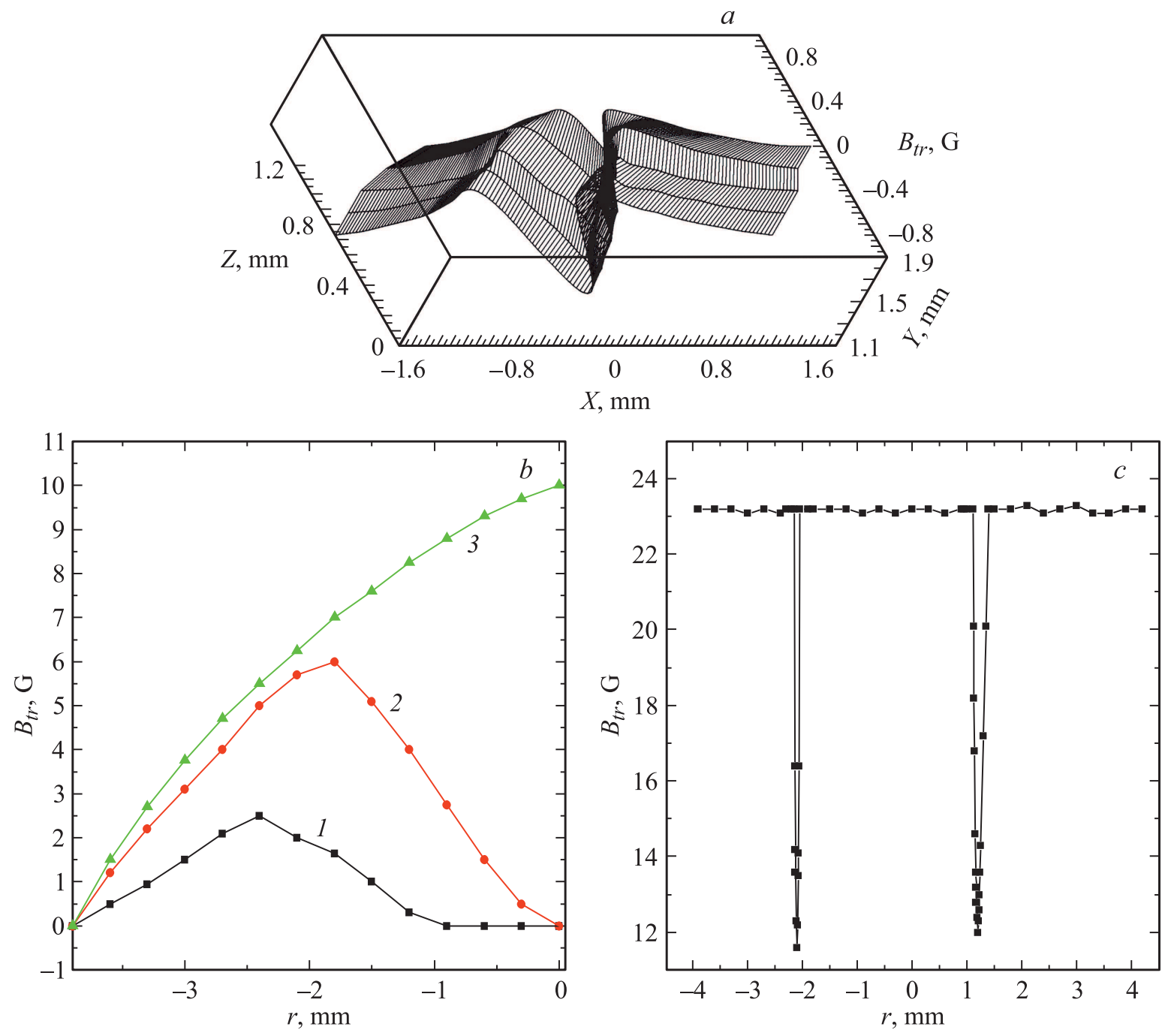

Рис. 3. Пространственное распределение плотности захваченного магнитного потока $B_{t r}(X, Y, Z)$ вокруг образцов: $a-$ образца № 1 для расстояния между образцом и $\Pi \mathrm{X} Z=50 \mu \mathrm{m}$, измеренное в режиме ZFCMFA в поле $100 \mathrm{G} ; b-$ образца № 2, измеренное в режиме ZFCMFA в магнитных полях $25 \mathrm{G}$ (кривая 1 ), $50 \mathrm{G}$ (кривая 2 ), $65 \mathrm{G}$ (кривая 3 ); $c$ - радиальное распределение $B_{t r}(r)$ для образца № 2, измеренное в режиме ZFCMFA и магнитном поле 0.13 Т. Температура $77.4 \mathrm{~K}$.

в слабых внешних магнитных полях, такие поля в режиме FC (field cooling) также захватываются YBCO и $\mathrm{Bi}_{2} \mathrm{Sr}_{2} \mathrm{CaCu}_{2} \mathrm{O}_{8+x}$ (BSCCO) монокристаллическими образцами. Следовательно, широкий низкополевой динамический диапазон был необходим, в частности, для прецизионного измерения первого джозефсоновского критического магнитного поля проникновения гипервихрей в керамических ВТСП [13] и термодинамических первых критических полей суб- и нанокристаллитов ВТСП образцов [7]. По уровню ЗМП оценивалась мейснеровская доля в ВТСП образцах. Слабые поля создавались питанием соленоида высокостабильным биполярным генератором разрядных токов, управляемым двоичным кодом [15]. Если токовые и потенциальные контакты ПХ подведены с боковых сторон подложки, то минимальный зазор между зеркально отполированной поверхностью образцов и ПХ может достигать $1 \mu \mathrm{m}$.
В случае использования промышленных ПХ, из-за токовых и потенциальных контактов минимальный зазор между рабочей поверхностью ПХ и поверхностью образца может составить до $200 \mu \mathrm{m}$. В этом случае для установления точного значения магнитного поля необходимо построить нормированное осевое распределение $B_{t r}(Z) / B_{t r}^{\max }(0)$ от координаты $Z$. Экстраполяция кривой к оси ординат, смещенной на $-200 \mu$ m по оси $Z$, показало, что потери сигнала на расстоянии $200 \mu \mathrm{m}$ от поверхности образцов составляют не более 5\% от $B_{t r}^{\max }(0)$. Таким образом, с помощью построения нормированных пространственных распределения плотности ЗМП по осям $X, Y, Z$, для площади объекта, большей чем эффективная площадь рабочей поверхности ПХ $S_{e f f}$, устанавливается истинная картина распределения магнитного поля $B(X, Y, Z)$ над образцом. В микроскопе был обеспечен коэффициент нелинейности менее $0.01 \%$ 

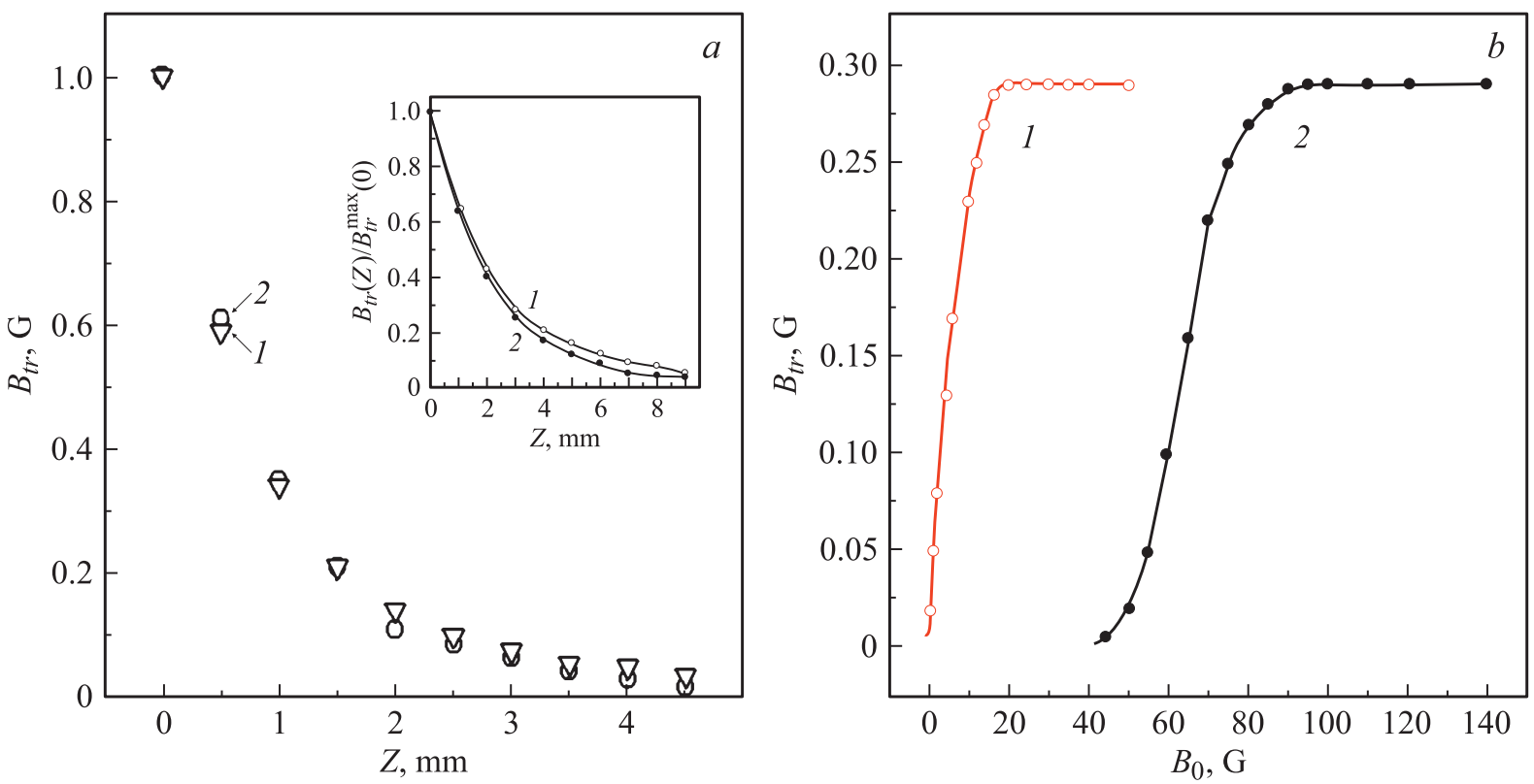

Рис. 4. $a$ - нормированное осевое распределение $B_{t r}(z) / B_{t r}^{\max }(0)$ для образца № 1, измеренное в режимах $\mathrm{FC}$ (кривая 1 ) и ZFC (кривая 2$) ; b-$ магнитополевые зависимости плотности ЗМП $B_{t r}\left(B_{0}\right)$ для образца № 3, измеренные в режимах $\mathrm{FC}$ (кривая 1$)$ и ZFCMFA (кривая 2). Температура $77.4 \mathrm{~K}$.

(который в основном задается нелинейностью ПХ) во всем динамическом диапазоне микроскопа вне зависимости от достигнутой высокой чувствительности и точности магнитометра. Для определения коэффициента нелинейности микроскопа через соленоид пропускался строго линейный нарастающий ток, и измерялась величина поля. По степени отклонения линейности тока и поля было установлено, что коэффициент нелинейности магнитометра выше в области слабых полей и уменьшается при росте поля, становясь меньше $0.01 \%$ при поле $B=10^{-2}$ Т. Такая высокая линейность обеспечивается как в режиме, когда датчик фиксирован и меняется поле, так и в режиме, когда поле фиксировано и идет сканирование образца по отношению к датчику.

\section{3. Результаты измерений и их обсуждение}

Магнитный поток, захваченный в участках с сильным пиннингом, замыкается не только вокруг ВТСП образца, но и через участки с более слабым пиннингом. Следовательно, на разных расстояниях от образца можно селективно измерить вклады в величину $B_{t r}(X, Y, Z)$ фрагментов плотностей магнитного потока, захваченных в разных структурных единицах образца. Для этого измерялись параметры плотности ЗМП и ее изменение с изменением топологии захваченного потока.

На рис. 3, а приведено пространственное распределение плотности ЗМП $B_{t r}(X, Y, Z)$ вблизи BSCCO монокристаллического образца размером $4 \times 4 \times 0.6 \mathrm{~mm}$ (образец № 1), измеренное в режиме ZFCMFA (zero field cooling with magnetic flux accumulation) [7] в поле $100 \mathrm{G}$ при температуре $77.4 \mathrm{~K}$ на расстоянии $50 \mu \mathrm{m}$ от поверхности образца. Координата $Z$ менялась в диапазоне $50 \mu \mathrm{m}-1 \mathrm{~mm}$. Как видно из рисунка, в середине образца зависимость проходит через нуль от минимума к максимуму. При удалении от поверхности образца ПХ постепенно перестает „замечать“ часть потока, захваченного в кристаллитах, которая через слабые связи замыкается внутри образца. Это приводит к спаду зависимости $B_{t r}(X, Y, Z)$. При приближении к краю образца силовые линии плотности ЗМП меняют свое направление и начинают замыкаться не внутри образца, а вокруг него.

На рис. $3, b$ приведено радиальное распределение $B_{t r}(r)$ для массивного текстурированного (ось $c$ перпендикулярна плоскости образца) образца ҮВСО в форме тонкого диска $D=8 \mathrm{~mm}, h \approx 0.7 \mathrm{~mm}$ (образец № 2), измеренное в режиме ZFCMFA при температуре $77.4 \mathrm{~K}$. Как видно из рис. $3, b$, граница применимости модели Бина ограничивается полем $65 \mathrm{G}$, которое примерно соответствует первому критическому магнитному полю проникновения магнитного потока в кристаллиты.

На рис. 3,c приведены радиальные распределения $B_{t r}(r)$ для образцов № 2, измеренные в режиме ZFCMFA в магнитном поле $0.13 \mathrm{~T}$ при температуре $77.4 \mathrm{~K}$. Для образца № 2 в области сильных полей подавляются междоменные слабые связи и отчетливо видны домены размерами $\sim 3-4 \mathrm{~mm}$ (использованные в установке ПХ имели площадь рабочей поверхности $S \sim 50 \times 100 \mu \mathrm{m})$.

На рис. 4, $a$ приведено нормированное осевое распределение $B_{t r}(z) / B_{t r}^{\max }(0)$ для образца № 1, измеренное 
в режимах $\mathrm{FC}$ (кривая 1) и ZFC (zero field cooling) (кривая 2) при температуре $77.4 \mathrm{~K}$. В режиме FC распределение ЗМП происходит более однородно, чем в режиме ZFC, поскольку исходное однородное внешнее поле более однородно захватывается после его снятия. Согласно рис. 4, $a$, спад кривых 1 и 2 происходит одинаково. Это показывает, что в монокристаллическом образце центры пиннинга имеют одинаковую глубину и распределены однородно. Для поликристаллического образца в случае однородной намагниченности зависимость $B_{t r}(z) / B_{t r}^{\max }(0)$ спадает медленнее, чем в случае неоднородной намагниченности. Таким образом, неоднородное пространственное распределение ЗМП в образце сильнее деформирует поле вокруг него.

На рис. $4, b$ для демонстрации чувствительности микроскопа и стабильности созданных низких полей приведены магнитополевые зависимости плотности ЗМП $\mathrm{Btr}(\mathrm{Ho})$ для $\mathrm{YBCO}$ монокристаллического образца размерами $\sim 1.2 \times 1.1 \times 0.02 \mathrm{~mm}$ (образец № 3), измеренные в режимах FC (кривая 1) и ZFCMFA (кривая 2). Согласно полученным результатам, приложение магнитного поля величиной $\sim 10^{-3} \mathrm{G}$ после снятия захватывается образцом. Это вызвано пиннингом джозефсоновских и абрикосовских вихрей на границах двойникования и других дефектах образца.

Учитывая, что осциллирующее затухающее во времени магнитное поле существенно уменьшает Seff. ПХ по сравнению с $S$ [16], использование ПХ с $S \sim 1 \times 1 \mu \mathrm{m}$ [9] открывает серьезные перспективы для применения холловских микроскопов в различных областях науки и техники [17].

\section{Конфликт интересов}

Автор заявляет, что у него нет конфликта интересов.

\section{Список литературы}

[1] Д.М. Гинзберг (ред.). Физические свойства высокотемпературных сверхпроводников. (Мир, М., 1990).

[2] Y. Syono, M. Kikuchi, K. Oh-Ishi, H. Hiraga, H. Arai, Y. Matsui, N. Kobayashi, T. Sasaoka, Y. Muto. Jap. J. Appl. Phys., 26 (4A), L498 (1987).

[3] G. Roth, D. Ewert, G. Heger, M. Hervieu, C. Michel, B. Raveau, F. D’Yvoire, A. Revcolevschi. Z. Phys. B, 69 (1), 21 (1987).

[4] S. Nakahara, T.Boone, M.F. Yan, G.J. Fisanick, D.M. Johnson Jr. J. Appl. Phys., 63 (2), 451 (1988).

[5] А.А. Абрикосов, А.И. Буздин, М.Л. Кулич, Д.А. Купцов. ЖЭТФ, 95, 371 (1989).

[6] Д. Нелсон, М. Уиттинхем, Т. Джордж (ред.). Высокотемпературные сверхпроводники. (Мир, М., 1988).

[7] Х.Р. Ростами. Письма в ЖЭТФ, 105, 754 (2017).

[8] R.C. Barrett, C.F. Quate. Rev. Sci. Instrum., 62, 1393 (1991).

[9] A. Oral, S.J. Bending, M. Henini. J. Vac. Sci. Technol. B, 14, 1202 (1996).

[10] V. Cambel, J. Fedor, D. Gregušová, P. Kováč, I. Hušek Supercond. Sci. Technol., 18 (4), 417 (2005).
[11] А.К. Игнатьев, А.А. Орлов, С.В. Перченко, Д.А. Станкевич. Письма в ЖТФ, 43 (15), 3 (2017).

[12] Х.Р. Ростами. ПТЭ, 2, 112 (2016).

[13] Э.Б. Сонин. Письма в ЖЭТФ, 47, 415 (1988).

[14] B. Loegei, D. Bolmont, A. Mehdaoui. Physica C, 159, 809 (1989).

[15] Х.P. Ростами. Преобразователь код-ток. Патент RU N2007862 C1,15.02.94., Бюл. № 3.

[16] Kh.R. Rostami, I.P. Nikitin. Measurement, 153, 107423 (2020).

[17] Яо Нан, Лин Ван Чжун (ред.). Справочник по микроскопии для нанотехнологии. (Научный мир, М., 2011). 